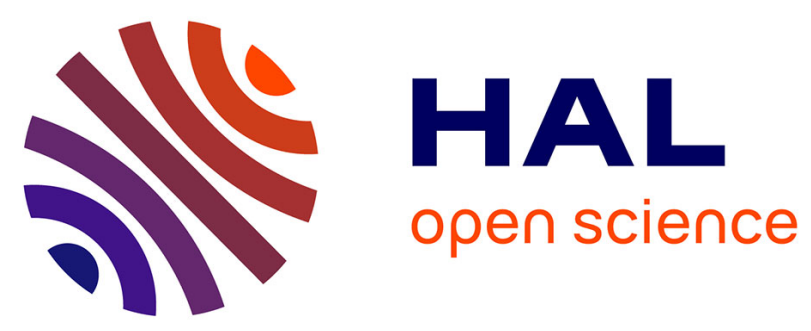

\title{
Intra-operative Update of Boundary Conditions for Patient-specific Surgical Simulation
}

Eleonora Tagliabue, Marco Piccinelli, Diego Dall'Alba, Juan Verde, Micha Pfeiffer, Riccardo Marin, Stefanie Speidel, Paolo Fiorini, Stéphane Cotin

\section{- To cite this version:}

Eleonora Tagliabue, Marco Piccinelli, Diego Dall'Alba, Juan Verde, Micha Pfeiffer, et al.. Intraoperative Update of Boundary Conditions for Patient-specific Surgical Simulation. MICCAI 2021 24th International Conference on Medical Image Computing and Computer Assisted Intervention, Sep 2021, Strasbourg, France. pp.373-382. hal-03315008

\section{HAL Id: hal-03315008 \\ https://hal.science/hal-03315008}

Submitted on 5 Aug 2021

HAL is a multi-disciplinary open access archive for the deposit and dissemination of scientific research documents, whether they are published or not. The documents may come from teaching and research institutions in France or abroad, or from public or private research centers.
L'archive ouverte pluridisciplinaire HAL, est destinée au dépôt et à la diffusion de documents scientifiques de niveau recherche, publiés ou non, émanant des établissements d'enseignement et de recherche français ou étrangers, des laboratoires publics ou privés. 


\title{
Intra-operative Update of Boundary Conditions for Patient-specific Surgical Simulation
}

\author{
Eleonora Tagliabue ${ }^{1}$, Marco Piccinelli ${ }^{1}$, Diego Dall'Alba ${ }^{1}$, Juan Verde $^{2}$, Micha \\ Pfeiffer $^{3}$, Riccardo Marin ${ }^{4}$, Stefanie Speidel ${ }^{3}$, Paolo Fiorini ${ }^{1}$, and Stéphane \\ $\operatorname{Cotin}^{5}$ \\ 1 University of Verona, Verona, Italy (eleonora.tagliabue@univr.it) \\ ${ }^{2}$ Institut de Chirurgie Guide par l'Image, Strasbourg, France \\ 3 National Center for Tumor Diseases, Dresden, Germany \\ 4 Sapienza University of Rome, Rome, Italy \\ 5 INRIA, Strasbourg, France
}

\begin{abstract}
Patient-specific Biomechanical Models (PBMs) can enhance computer assisted surgical procedures with critical information. Although pre-operative data allow to parametrize such PBMs based on each patient's properties, they are not able to fully characterize them. In particular, simulation boundary conditions cannot be determined from preoperative modalities, but their correct definition is essential to improve the PBM predictive capability. In this work, we introduce a pipeline that provides an up-to-date estimate of boundary conditions, starting from the pre-operative model of patient anatomy and the displacement undergone by points visible from an intra-operative vision sensor. The presented pipeline is experimentally validated in realistic conditions on an ex vivo pararenal fat tissue manipulation. We demonstrate its capability to update a PBM reaching clinically acceptable performances, both in terms of accuracy and intra-operative time constraints.
\end{abstract}

Keywords: Intra-operative model update $\cdot$ Boundary conditions $\cdot$ Biomechanical modeling

\section{Introduction}

An up-to-date Patient-specific Biomechanical Model (PBM) of the surgical scenario can bring benefits to the surgical practice in several ways. In computerassisted interventions, such PBM can guide surgeons towards the structures of interest, which might be hidden from the partial view available intra-operatively [11]. A further application is in the field of autonomous robotic surgery, where a $\mathrm{PBM}$ of the current surgical condition is required for verification of the robotic actions in a controlled environment, before execution [5]. Moreover, PBMs can play the role of virtual sensors to estimate interaction forces between instruments and tissues when direct force measurement systems are not available, and provide a feedback to the surgeon [8]. The predictive power of such PBMs highly relies on their accurate parametrization, which has to be tailored to each new 
patient. Patient-specific geometry and tissues' elastic properties can be extracted from pre-operative anatomical images or using ad-hoc modalities such as elastographic techniques, allowing to build a PBM with personalized properties [7]. However, information available before the intervention is often insufficient to fully characterize PBMs to the extent required to achieve clinically accepted accuracy. In particular, there is usually no way to delineate the adhesions between neighboring organs from pre-operative data. Such adhesions define simulation Boundary Conditions (BCs), thus they are key to obtain an accurate model [12, $15,13]$. As a consequence, reliable BCs can be estimated only from data that are collected intra-operatively $[15,13,11]$.

The problem of intra-operative estimation of BCs has been tackled by only few works. In $[18,13]$, the position of BCs is initialized based on statistical atlases, leading to a method which is not robust to inter-patient variations. Other approaches [14] propose to update BCs exploiting additional intra-operative sensors, undermining their direct applicability to the standard clinical practice. In $[15,13]$, authors propose to use stochastic filters to estimate the elasticity of the hepatic ligaments, exploiting intra-operative observations of the tissue state. However, the filters' inference time strongly depends on their parameters initialization, which is highly sensitive to each patient's properties, possibly introducing a degradation in the performances from case to case.

A recent research trend has focused on the usage of Deep Neural Networks (DNNs) to update a biomechanical model based on intra-operative data $[10,4$, $17,16,19]$. These works have shown that DNNs can learn biomechanical models even when trained with synthetic data only, while guaranteeing very low inference time. Furthermore, Pfeiffer et al. $[17,16]$ demonstrated that DNNs can also learn a surface representation, thus being able to handle any input geometric model. However, all these works have focused on the estimation of the full 3D displacement field to accomplish the task of intra-operative registration, either without taking advantage of any PBM parametrization inferred from preoperative data or assuming that BCs are fixed and a-priori known.

In this work, we present a complete pipeline that allows to continuously update an existing PBM by estimating model BCs, starting directly from the raw intra-operative point cloud of the deforming anatomy provided by a vision sensor and without relying on any a-priori assumption about their location. We conduct an experimental validation of the complete framework on an ex vivo human model, including the anterior renal fascia (Gerota's fascia), the pararenal adipose tissue and the kidney. The manipulation of these tissues is a key step during most of surgical kidney procedures (most importantly during partial nephrectomy). Obtained results demonstrate that the proposed pipeline is able to update a real PBM, respecting clinically acceptable requirements both in terms of accuracy and timing, thus allowing to account for possible intra-operative changes of the BCs caused by surgical manipulations. 


\section{Method}

In order to provide an up-to-date PBM that continuously follows the current surgical scenario, we rely on a framework involving two independent processes which run concurrently. The first process is entirely dedicated to a physics-based simulation of the surgical environment capable of real-time performances [24]. Such simulation leverages the PBM created from pre-operative data, characterized by both the undeformed 3D geometry of the anatomy and its known mechanical properties. The second process is devoted to a strategy for updating PBM parametrization during the intervention, starting from intra-operative sensor data. In this work, we focus on this second task. In particular, we present a pipeline to update simulation BCs from the 3D point cloud of the surgical scene with a very short latency ${ }^{1}$. This allows the simulation to continuously reflect the changes introduced in the environment by surgical manipulations (Fig. 1).

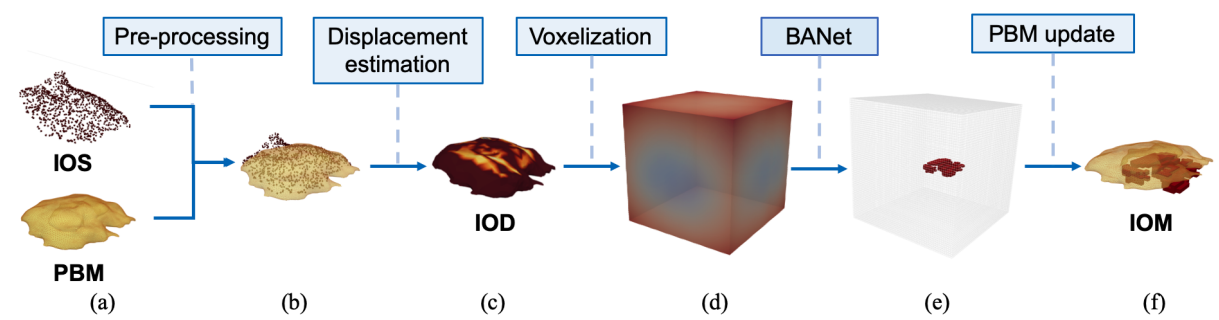

Fig. 1. Overview of the pipeline to update PBM boundary conditions. (a) Initial data: IOS and PBM; (b) rigidly aligned data; (c) estimated IOD, where brighter color is associated with highest displacement; (d) voxelized representation, where grid cells are colored based on the signed distance field from PBM surface; (e) estimated BCs in grid space; (f) PBM annotated with estimated BCs, giving the intra-operative model IOM.

Pre-processing The acquired raw 3D point cloud passes through an initial pre-processing step. First, both color and spatial segmentation are performed to extract the current view of the deformed Intra-Operative Surface (IOS) from the full anatomical point cloud. The extracted IOS is then rigidly aligned to its corresponding portion of the PBM, based on geometric features and known spatial relations estimated at the beginning of surgery.

Displacement estimation We calculate the Intra-Operative Displacement field (IOD) which maps each point in the IOS to its corresponding one on the undeformed PBM. To achieve this, we estimate a correspondence with a nearestneighbor pairing between the point cloud and the PBM, and we refine it using

\footnotetext{
${ }^{1}$ Project available at https://gitlab.com/altairLab/banet
} 
the ZoomOut method [9]. This non-rigid approach is entirely intrinsic, promotes isometric solutions (i.e., correspondences that preserve the surface distances between the points), and has approximately linear complexity. Thus, ZoomOut guarantees a trade-off between accuracy and timing, making it ideal for realtime precision surgical operations, where we assume the folding and bending of the surface preserves surface geometry (i.e., boundaries and metric) without introducing dramatic stretching.

Voxelization In order to exploit convolutional filters, input information is converted into a grid-like volume of dimension $64 \times 64 \times 64$ and side length $30 \mathrm{~cm}$. The PBM is encoded into the grid using its signed distance field and the IOD through a Gaussian interpolation.

BANet The boundary condition update is performed by BANet, a DNN estimating at which points a given deformable tissue is attached to the surrounding environment [22]. BANet has been validated on phantom data with simple geometry, but has never been applied to a real PBM and within a realistic clinical situation. BANet is a U-shaped network, which consists of an encoding and a decoding path. The former contains four stages of downsampling, each reducing the spatial resolution by half, resulting in a bottleneck layer which is $4^{3}$ grid cells in size. This allows information to travel across the spatial domain. Additional skip-connections enable the network to carry fine detail forward where necessary. To ensure a high inference speed, downsampling and upsampling are done via simple MaxPool and interpolation functions. The network is trained to approximate the function $f(P B M, I O D)=A P$, where $A P$ is the binary voxel field representation of the attachment points. The training dataset is composed of synthetic samples representing adipose tissues PBMs (with different random geometries and mechanical parameters) and annotated with randomly extracted BCs. In this work, we use the publicly available implementation of BANet with the provided pre-trained weights [22].

PBM update Finally, BANet-estimated BCs are mapped from grid space to the original PBM space, giving the Intra-Operative Model (IOM). This step completes the proposed pipeline, and the obtained IOM is used to update the intra-operative simulation running in the synchronous process.

\section{Experiments and Results}

Validation of the presented method is carried out on ex vivo pararenal fat tissue manipulation (Fig. 2a). Tissue's PBM is initialized with the specimen 3D geometry, generated from manual segmentation of its CT scan, and discretized with 65,538 tetrahedral elements. Its biomechanical properties are selected to be aligned with those observed for adipose tissues (i.e., St Venant Kirchhoff material 


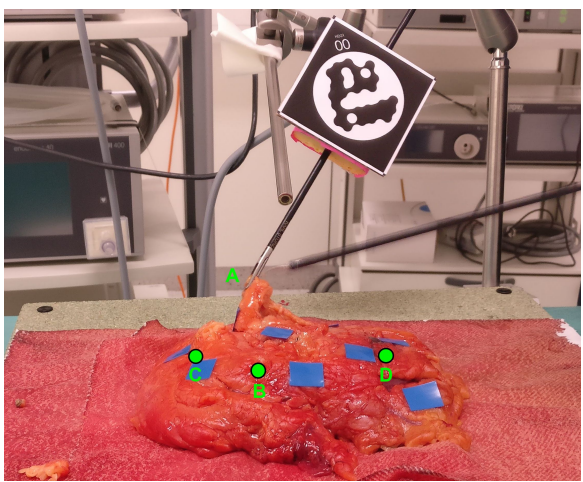

(a)

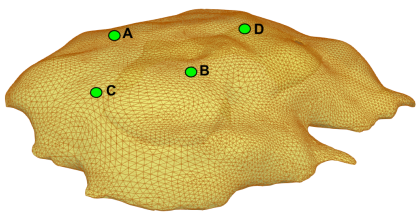

(b)

Fig. 2. (a) An example tissue manipulation. The surgeon manipulates the pararenal fat and anterior fascia with a laparoscopic instrument, grasping from point A. Point clouds of the tissue state are acquired with an RGB-D camera capturing the scene from the same perspective of the picture. (b) The PBM of the ex-vivo perinephric tissue. The position of the grasping points is marked with a letter and a green circle.

with Young's modulus $3 \mathrm{kPa}$ and Poisson ratio 0.45) [1]. Leveraging on the constructed PBM (Fig. 2b), it has been also possible to generate a synthetic dataset based on a real anatomical model. Such dataset allows us to evaluate method performances within a scenario which is influenced neither by sensor noise nor by inaccuracies introduced by ZoomOut, since the IOD can be estimated by directly matching corresponding points in the deformed and the undeformed configurations.

The capability of the method to update the PBM such that the virtual environment resembles the current tissue condition is assessed by comparing the deformed state in the simulated environment with the available ground truth deformed configuration. The deformed state in the virtual environment is obtained by performing a finite element (FE) simulation where model BCs are defined by the proposed approach and computing the Root Mean Squared Error (RMSE) between the simulated and the ground truth configurations. A stateof-the-art direct solver [20] is used together with an iterative Newton-Raphson method to solve the non-linear system of equations in the static domain, within the open-source SOFA framework [6]. The obtained simulation result is weakly sensitive to possible inaccuracies in the selected mechanical parameters, since the driving input to the simulation is represented by a displacement (i.e., the same displacement which is applied to the corresponding ground truth configuration) $[12]$.

\subsection{Synthetic adipose tissue manipulation}

A synthetic dataset of 600 samples of adipose tissue manipulation is generated following the same training data generation strategy used in [22], but keeping 
the considered PBM fixed (Fig. 2b). The median RMSE (interquartile range IQR) calculated on all the 3D model points between the deformed IOM and the corresponding reference sample is $1.4(0.7-3.2) \mathrm{mm}$, indicating an overall good matching (Fig. 3a). Since ground truth BCs are available for the synthetic dataset, we further assess prediction accuracy by computing the Dice coefficient (DSC) [23], which measures the overlapping area between ground truth and predicted BCs. Median (IQR) value for DSC is $0.51(0.40-0.60)$. Fig. 3b and c show that the network is challenged by the complex geometry and fails to accurately identify BCs when they are distributed along the sharp edges of the mesh, especially when the amount of visible surface is very small and does not capture the region undergoing the highest deformation. However, when the visible surface captures the region with greatest deformation (Fig. 3b), BANet is able to provide a plausible prediction, which leads to a precise matching between the simulated and the reference configuration.

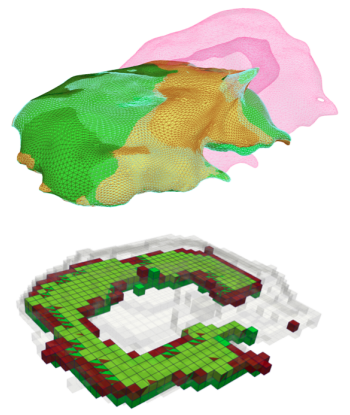

(a)

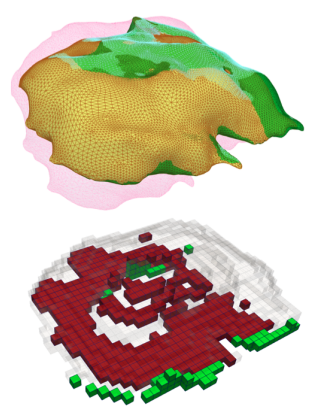

(b)

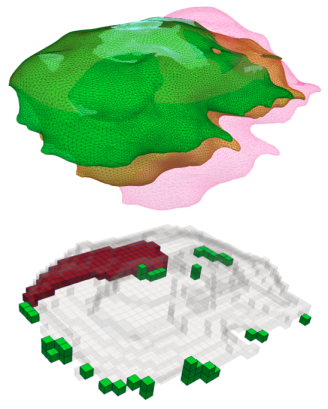

(c)

Fig. 3. Results on the synthetic dataset considering input displacements above $25 \mathrm{~mm}$. Upper row: ground truth deformed configuration (green) and simulated deformed configuration obtained when using predicted BCs (yellow), with the considered visible surface (light blue). Pink mesh represents the deformed configuration when BCs are unknown, thus undefined. Lower row: ground truth BCs (green) and predicted BCs (red) in grid space. (a) Good overlap between simulated and ground truth configurations $(\mathrm{RMSE}=1.6 \mathrm{~mm})$, due to good prediction accuracy ( $\mathrm{DSC}=0.68)$. (b) Good overlap ( $\mathrm{RMSE}=1.7 \mathrm{~mm}$ ) is possible even if prediction is not the same as the ground truth one $(\mathrm{DSC}=0.09)$. (c) High RMSE $(14.6 \mathrm{~mm})$ is due to an inaccurate prediction $(\mathrm{DSC}=0.02)$, when visible surface does not provide enough information about tissue state.

\subsection{Real adipose tissue manipulation}

The presented pipeline is employed to update the PBM during ex vivo pararenal tissue manipulation. An expert surgeon is asked to grasp the human tissue with a laparoscopic tool from four pre-defined points, whose position is known in 
the 3D model space thanks to CT-visible markers, and lift it to the maximum reachable extent that prevents tissue tearing (Fig. 2a). After pulling the tissue from all the points, the surgeon introduces a change in BCs by dissecting some tissue adhesions, and repeats the acquisitions. Acquired data are relative to three different initial states (i.e., two dissection stages); however, it has not been always possible to lift from all the grasping points due to excessive tissue damage introduced by dissection. Intra-operative point clouds capturing deformed tissue states are acquired through an Intel RealSense D435 RGB-D camera. Collected images allow to extract the displacement applied to the tissue by the surgeon, by tracking a STag marker attached to the instrument (Fig. 2a) [3]. To evaluate the presented method, point clouds at regular lifting steps of $10 \mathrm{~mm}$ are extracted and passed through the complete pipeline described in Section 2. As soon as a new estimate of $\mathrm{BCs}$ is available, we perform a FE simulation to the obtained IOM applying the same input displacement applied by the surgeon. We then calculate the RMSE between each point in the acquired point cloud and its corresponding one, as estimated by ZoomOut, in the deformed IOM.

Table 1 reports the average RMSE over 10 runs of the whole pipeline, relative to the error at rest (average $3.62 \mathrm{~mm}$, which includes contributions of segmentation and registration errors) at increasing pulling levels. The reason why we report results over multiple runs is that we rely on an approximate nearest neighbour for ZoomOut to minimize the computational overhead, which might however introduce some differences between different runs. The average time required to update the synchronous PBM simulation is $1.44 \pm 0.14 s$ (with an average of $0.039 s$ dedicated to pre-processing, $1.39 s$ to displacement estimation, and $0.013 s$ to BANet), tested on a workstation with an AMD Ryzen7 3700X CPU and NVIDIA RTX 2070 SUPER graphics card. This update rate allows to provide feedback to the operating surgeon and/or the autonomous system based on a model reflecting the changes in clinical settings with a latency compatible with standard surgical workflows. Average RMSEs remain below $5 \mathrm{~mm}$ in almost all the cases, which is aligned with the accuracy levels required for model-guided intra-operative applications in the context of minimally-invasive surgery $[16,4,11]$. Furthermore, obtained RMSEs are significantly lower than the ones achieved when BCs are unknown, thus they cannot be defined and the simulation remains unconstrained (last column in the table). In general, RMSE increases with increasing deformation. This might be due to the fact that the high deformations cause poor surface estimation from [21], which assumes that the point cloud already represents the desired surface without noise or topological artifacts. In such case, ZoomOut promotes a disturbed correspondence (i.e., isometric to a wrong surface). To improve this step, investigating the point cloud denoising techniques seems a promising future direction. This further motivates the worse RMSE values obtained in correspondence to grasping point $\mathrm{B}$, whose point clouds are partially occluded by the surgical tool. 
Table 1. RMSE between ground truth point clouds and their corresponding points in the virtual environment when using predicted BCs, at increasing deformation levels $[\mathrm{mm}]$. Acquisitions are grouped by grasping point (A, B, C, D) and initial tissue state $(1,2,3)$. Reported errors are relative to the error at rest and represent the average over 10 runs of the entire pipeline. Missing values are due to failures in instrument tracking. Last column reports the RMSE obtained when no BCs are defined in the virtual environment $\left(\operatorname{Mean}_{U C}\right)$.

\begin{tabular}{c|c|c|c|c|c|c|c|c|c|} 
Grasp & State & $10 \mathrm{~mm}$ & $20 \mathrm{~mm}$ & $30 \mathrm{~mm}$ & $40 \mathrm{~mm}$ & $50 \mathrm{~mm}$ & Mean & Std & Mean $_{U C}$ \\
\hline \multirow{2}{*}{ A } & 1 & - & 0.94 & 1.06 & 1.28 & 6.81 & 2.52 & 2.48 & 24.93 \\
& 2 & - & - & 1.42 & 2.42 & 2.75 & 2.20 & 0.65 & 31.99 \\
\hline \multirow{3}{*}{ B } & 1 & 2.96 & 6.75 & - & - & - & 4.86 & 1.90 & 13.02 \\
& 2 & 5.05 & 9.91 & 11.47 & - & - & 8.81 & 2.84 & 27.06 \\
& 3 & 3.27 & 11.71 & 9.12 & 3.92 & - & 7.01 & 3.60 & 31.53 \\
\hline \multirow{3}{*}{ C } & 1 & - & 1.65 & 2.49 & - & - & 2.07 & 0.42 & 18.66 \\
& 2 & 5.30 & 2.54 & 3.84 & - & - & 3.89 & 1.42 & 14.23 \\
& 3 & 2.59 & 4.61 & 6.93 & 10.76 & - & 6.22 & 3.04 & 18.79 \\
\hline & 1 & 1.07 & - & - & - & - & 1.07 & 0.00 & 7.72 \\
D & 2 & - & 1.32 & 1.57 & 3.98 & 5.25 & 3.03 & 1.71 & 19.76 \\
& 3 & 1.51 & 2.28 & 3.57 & - & - & 2.45 & 0.85 & 7.29 \\
\hline Mean & & 3.11 & 4.64 & 4.61 & 4.47 & 4.94 & & & \\
Std & & 1.50 & 3.90 & 3.52 & 3.32 & 1.80 & & & \\
\hline
\end{tabular}

\section{Discussion and Conclusion}

In this work, we have presented a complete pipeline that allows to update a patient-specific pre-operative model for surgical assistance, based on data acquired during the intervention. Validation experiments have shown that the presented pipeline can be used to successfully update a PBM exploiting data coming directly from intra-operative sensors, while respecting both accuracy and time constraints compatible with standard minimally-invasive surgical applications. The quality of the final result is influenced by the different sources of errors that are introduced throughout the various stages of the pipeline, from an imprecise initial rigid alignment, to the presence of sensor noise and inaccurate computation of corresponding points. In future works, we plan to improve the pre-processing stage, for instance by reconstructing the point cloud from the stereo-endoscope view [2]. Inaccurate surface matching can be addressed by either letting the network implicitly solve the surface correspondence problem as in [16], by providing salient points extracted from camera view to ZoomOut, or by improving surface estimation [25]. In particular, relying on DNN to directly solve for surface correspondences seems promising to further improve the time performances of the current implementation, where ZoomOut is responsible of the main computational overhead. By providing an update of model BCs with a very short delay, our method could handle situations with dynamically changing BCs, for example involving dissection, sutures removal or topological changes. 
However, due to the fact that the surgical environment is intrinsically evolving in time and the network has shown to benefit from the availability of more informative input data, we expect that the robustness of the prediction will be improved by considering time dynamics and we will tackle this in future works.

Acknowledgements Authors would like to thank the preclinical research staff at IHU Strasbourg for their assistance and support during the experiments. This project has received funding from the European Research Council (ERC) under the European Union's Horizon 2020 research and innovation programme (grant agreement No. 742671 "ARS"), from French state funds managed within the "Plan Investissements dAvenir" and from the ANR (reference ANR-10-IAHU$02)$.

\section{References}

1. Alkhouli, N., Mansfield, J., Green, E., Bell, J., Knight, B., Liversedge, N., Tham, J.C., Welbourn, R., Shore, A.C., Kos, K., et al.: The mechanical properties of human adipose tissues and their relationships to the structure and composition of the extracellular matrix. American Journal of Physiology-Endocrinology and Metabolism 305(12), E1427-E1435 (2013)

2. Allan, M., Mcleod, J., Wang, C.C., Rosenthal, J.C., Fu, K.X., Zeffiro, T., Xia, W., Zhanshi, Z., Luo, H., Zhang, X., et al.: Stereo correspondence and reconstruction of endoscopic data challenge. arXiv preprint arXiv:2101.01133 (2021)

3. Benligiray, B., Topal, C., Akinlar, C.: Stag: A stable fiducial marker system. Image and Vision Computing 89, 158-169 (2019)

4. Brunet, J.N., Mendizabal, A., Petit, A., Golse, N., Vibert, E., Cotin, S.: Physicsbased deep neural network for augmented reality during liver surgery. In: International Conference on Medical image computing and computer-assisted intervention. pp. 137-145. Springer (2019)

5. Choi, H., Crump, C., Duriez, C., Elmquist, A., Hager, G., Han, D., Hearl, F., Hodgins, J., Jain, A., Leve, F., et al.: On the use of simulation in robotics: Opportunities, challenges, and suggestions for moving forward. Proceedings of the National Academy of Sciences 118(1) (2021)

6. Faure, F., Duriez, C., Delingette, H., Allard, J., Gilles, B., Marchesseau, S., Talbot, H., Courtecuisse, H., Bousquet, G., Peterlik, I., et al.: Sofa: A multi-model framework for interactive physical simulation. In: Soft tissue biomechanical modeling for computer assisted surgery, pp. 283-321. Springer (2012)

7. Galbusera, F., Cina, A., Panico, M., Albano, D., Messina, C.: Image-based biomechanical models of the musculoskeletal system. European radiology experimental 4(1), 1-13 (2020)

8. Haouchine, N., Kuang, W., Cotin, S., Yip, M.: Vision-based force feedback estimation for robot-assisted surgery using instrument-constrained biomechanical threedimensional maps. IEEE Robotics and Automation Letters 3(3), 2160-2165 (2018). https://doi.org/10.1109/LRA.2018.2810948

9. Melzi, S., Ren, J., Rodolà, E., Sharma, A., Wonka, P., Ovsjanikov, M.: Zoomout: spectral upsampling for efficient shape correspondence. ACM Transactions on Graphics (TOG) 38(6), 155 (2019) 
10. Mendizabal, A., Tagliabue, E., Brunet, J.N., DallAlba, D., Fiorini, P., Cotin, S.: Physics-based deep neural network for real-time lesion tracking in ultrasoundguided breast biopsy. In: Computational Biomechanics for Medicine, pp. 33-45. Springer (2019)

11. Mendizabal, A., Tagliabue, E., Hoellinger, T., Brunet, J.N., Nikolaev, S., Cotin, S.: Data-driven simulation for augmented surgery. In: Developments and Novel Approaches in Biomechanics and Metamaterials, vol. 132, pp. 71-96. Springer (Jul 2020). https://doi.org/10.1007/978-3-030-50464-9

12. Miller, K., Lu, J.: On the prospect of patient-specific biomechanics without patientspecific properties of tissues. Journal of the mechanical behavior of biomedical materials 27, 154-166 (2013)

13. Nikolaev, S., Cotin, S.: Estimation of boundary conditions for patient-specific liver simulation during augmented surgery. International Journal of Computer Assisted Radiology and Surgery (2020)

14. Peterlik, I., Courtecuisse, H., Duriez, C., Cotin, S.: Model-based identification of anatomical boundary conditions in living tissues. In: International Conference on Information Processing in Computer-Assisted Interventions. pp. 196-205. Springer (2014)

15. Peterlik, I., Haouchine, N., Ručka, L., Cotin, S.: Image-driven stochastic identification of boundary conditions for predictive simulation. In: International Conference on Medical Image Computing and Computer-Assisted Intervention. pp. 548-556. Springer (2017)

16. Pfeiffer, M., Riediger, C., Leger, S., Kühn, J.P., Seppelt, D., Hoffmann, R.T., Weitz, J., Speidel, S.: Non-rigid volume to surface registration using a data-driven biomechanical model. In: International Conference on Medical Image Computing and Computer-Assisted Intervention. Springer (2020)

17. Pfeiffer, M., Riediger, C., Weitz, J., Speidel, S.: Learning soft tissue behavior of organs for surgical navigation with convolutional neural networks. International Journal of Computer Assisted Radiology and Surgery pp. 1-9 (2019)

18. Plantefève, R., Peterlik, I., Haouchine, N., Cotin, S.: Patient-specific biomechanical modeling for guidance during minimally-invasive hepatic surgery. Annals of biomedical engineering 44(1), 139-153 (2016)

19. Saeed, S.U., Taylor, Z.A., Pinnock, M.A., Emberton, M., Barratt, D.C., Hu, Y.: Prostate motion modelling using biomechanically-trained deep neural networks on unstructured nodes. In: International Conference on Medical Image Computing and Computer-Assisted Intervention. pp. 650-659. Springer (2020)

20. Schenk, O., Gärtner, K.: Solving unsymmetric sparse systems of linear equations with Pardiso. Future Generation Computer Systems 20(3), 475-487 (2004)

21. Sharp, N., Crane, K.: A laplacian for nonmanifold triangle meshes. In: Computer Graphics Forum. vol. 39, pp. 69-80. Wiley Online Library (2020)

22. Tagliabue, E., Dall'Alba, D., Pfeiffer, M., Piccinelli, M., Marin, R., Castellani, U., Speidel, S., Fiorini, P.: Data-driven intra-operative estimation of anatomical attachments for autonomous tissue dissection. IEEE Robotics and Automation Letters (2021)

23. Taha, A.A., Hanbury, A.: Metrics for evaluating 3d medical image segmentation: analysis, selection, and tool. BMC medical imaging 15(1), 29 (2015)

24. Taylor, Z.A., Cheng, M., Ourselin, S.: High-speed nonlinear finite element analysis for surgical simulation using graphics processing units. IEEE transactions on medical imaging 27(5), 650-663 (2008) 
25. Williams, F., Schneider, T., Silva, C., Zorin, D., Bruna, J., Panozzo, D.: Deep geometric prior for surface reconstruction. In: Proceedings of the IEEE/CVF Conference on Computer Vision and Pattern Recognition (CVPR) (June 2019) 